\title{
Constraints on Yukawa parameters by double pulsars
}

\author{
Xue-Mei Deng ${ }^{1,3}$, Yi Xie ${ }^{2,3}$ and Tian-Yi Huang ${ }^{2}$ \\ ${ }^{1}$ Purple Mountain Observatory, Chinese Academy of Sciences, Nanjing 210008, China \\ email: xmd@pmo.ac.cn \\ ${ }^{2}$ Department of Astronomy, Nanjing University, Nanjing 210093, China \\ ${ }^{3}$ Key Laboratory of Modern Astronomy and Astrophysics, Nanjing University, Ministry of \\ Education, Nanjing 210093, China
}

\begin{abstract}
Although Einstein's general relativity has passed all the tests so far, alternative theories are still required for deeper understanding of the nature of gravity. Double pulsars provide us a significant opportunity to test them. In order to probe some modified gravities which try to explain some astrophysical phenomena without dark matter, we use periastron advance $\dot{\omega}$ of four binary pulsars (PSR B1913+16, PSR B1534+12, PSR J0737-3039 and PSR B2127+11C) to constrain their Yukawa parameters: $\lambda=(3.97 \pm 0.01) \times 10^{8} \mathrm{~m}$ and $\alpha=(2.40 \pm 0.02) \times 10^{-8}$. It might help us to distinguish different gravity theories and get closer to the new physics.
\end{abstract}

Keywords. gravitation, relativity, pulsars: PSR B1913+16, PSR B1534+12, PSR J0737-3039, PSR B2127+11C

\section{Introduction}

The possible gravity-like "fifth" fundamental force in macroscopic scale suggested by Fischbach et al. (1986) evokes interest in many theories which intend to unify gravity with other known forces. The presence of this fifth force could be detected by searching for apparent deviations from Newtonian gravity. For instance, the fifth force would arise from the exchange of a new ultra-light boson which coupled to ordinary matter with a strength comparable to gravity. Typically, through adding a hypothetical Yukawa force to the Newtonian potential, this modified potential per mass takes the form:

$$
U(r)=-\frac{\mathcal{G} M}{r}\left(1+\alpha e^{-r / \lambda}\right),
$$

where $\alpha$ represents the strength of the Yukawa coupling, and $\lambda$ represents its length scale. Quite a number of works, such as Anderson et al. (1998), Fischbach \& Talmadge (1999), Reynaud \& Jaekel (2005), Sealfon et al. (2005), Brownstein \& Moffat (2006) and Iorio (2007), have been done to constrain these two parameters in astronomical scales.

Since the discovery of the binary pulsar PSR B1913+16 by Hulse \& Taylor (1975), binary pulsars promise an unprecedented opportunity to measure the effects of relativistic gravitation - see Stairs (2004), Lorimer (2005) for a review. For example, pulsar timing has provided indirect evidence for the existence of gravitational waves (Detweiler 1979), the binary pulsars data can constrain the existence of massive black hole binaries (Lommen \& Backer 2001), and the binary pulsars can also test the effects of strong relativistic internal gravitational fields on orbital dynamics (Bell et al. 1996). In addition, binary pulsars could help us to test various gravity theories. By fitting the arrival time of pulsars, observational parameters of binary pulsar are obtained in high precision. It worthy of noted that the periastron advance for binary pulsars could reach several degrees per 
year, which is about $10^{5}$ more than the perihelion advance of Mercury. Hence, the relativistic effects from binary pulsar are more remarkable than other celestial systems. In this paper we chose four best studied pulsar binaries: PSR B1913+16, PSR B1534+12, PSR J0737-3039, and PSR B2127+11C. We mainly focus on $\dot{\omega}$ of these four binaries data to constrain the Yukawa parameters.

\section{Secular periastron precession with fifth force}

When we consider only two body $\left(M_{1}\right.$ and $\left.M_{2}\right)$, the equations of motion of a point-mass binary with fifth force up to first order post-Newtonian approximation yields

$$
\ddot{\boldsymbol{r}}=\boldsymbol{a}_{\mathrm{N}}+\boldsymbol{a}_{1 \mathrm{PN}}
$$

where

$$
\begin{aligned}
\boldsymbol{a}_{\mathrm{N}}= & -\frac{\mathcal{G} M}{r^{2}}\left[1+\alpha\left(1+\frac{r}{\lambda}\right) \exp \left(-\frac{r}{\lambda}\right)\right] \boldsymbol{n}, \\
\boldsymbol{a}_{1 \mathrm{PN}}= & -\frac{\mathcal{G} M}{c^{2} r^{2}}\left\{\left[(\gamma+3 \nu) v^{2}-2(\gamma+\beta+\nu) \frac{\mathcal{G} M}{r}-\frac{3}{2} \nu \dot{r}^{2}\right] \boldsymbol{n}\right. \\
& -2(1+\gamma-\nu) \dot{r} \boldsymbol{v}\},
\end{aligned}
$$

where $M=M_{1}+M_{2}, \dot{r}=\left(n_{12} v_{12}\right), \boldsymbol{v}=\boldsymbol{v}_{1}-\boldsymbol{v}_{2}, \nu=M_{1} M_{2} / M^{2}, \boldsymbol{r}=\boldsymbol{r}_{12}, \boldsymbol{n}=\boldsymbol{n}_{12}$ and $\boldsymbol{a}=\mathrm{d} \boldsymbol{v}_{12} / \mathrm{d} t$. By the aid of the averaging method (Kozai, 1959), the secular periastron advance for a binary pulsar in $1 \mathrm{PN}$ is

$$
\frac{\mathrm{d} \omega}{\mathrm{d} t}=\frac{1}{2} \frac{n \alpha p^{2}}{\lambda^{2}} e^{-p / \lambda}+(2+2 \gamma-\beta) \frac{\mathcal{G} M n}{c^{2} p},
$$

where $n^{2} a^{3}=\mathcal{G} M, p=a\left(1-e^{2}\right), a$ is the semi-major axis and $e$ is the eccentricity of the binary. The periastron shift caused by Yukawa force is

$$
\left.\frac{\mathrm{d} \omega}{\mathrm{d} t}\right|_{\text {Yukawa }}=\frac{1}{2} \frac{n \alpha p^{2}}{\lambda^{2}} e^{-p / \lambda},
$$

which is different from the result given by (Iorio 2008). In this paper, we keep the angular momentum in the process of using the averaging method instead of approximate treatments such as $\exp (-r / \lambda) \approx 1-r / \lambda$ (Iorio 2008) to calculate the $\dot{\omega}$.

\section{Constraints on the Yukawa parameters}

Based on the same method of Damour \& Esposito-Farèse (1996), we will constrain the Yukawa parameters in $1 \sigma$ confidence level. Each set of binary data leads to a reduced $\chi^{2}: \chi_{\text {binary }}^{2}(\alpha, \lambda)=\left(\dot{\omega}_{\text {theory }}-\dot{\omega}_{\text {obs }}\right)^{2} / \sigma_{\text {obs }}^{2}$. To combine the constraints on $\alpha$ and $\lambda$ coming from different double pulsars systems, we add their individual $\chi^{2}$ as if they were part of a total experiment with uncorrelated Gaussian errors : $\chi_{\text {total }}^{2}(\alpha, \lambda)=\chi_{1913+16}^{2}(\alpha, \lambda)+$ $\chi_{1534+12}^{2}(\alpha, \lambda)+\chi_{0737-3039}^{2}(\alpha, \lambda)+\chi_{2127+11 C}^{2}(\alpha, \lambda)$. Therefore, the contour level $\Delta \chi_{\text {total }}^{2}(\alpha, \lambda)=2.3, \Delta \chi_{\text {total }}^{2}(\alpha, \lambda)=6.17$ and $\Delta \chi_{\text {total }}^{2}(\alpha, \lambda)=11.8$, where $\Delta \chi_{\text {total }}^{2}(\alpha, \lambda)=$ $\chi_{\text {total }}^{2}(\alpha, \lambda)-\left(\chi_{\text {total }}^{2}(\alpha, \lambda)\right)_{\text {min }}$, define respectively for two degrees of freedom the $68.3 \%$, $95.4 \%$ and $99.73 \%$ confidence levels. The $1 \sigma$ fit values for the Yukawa parameters are $\lambda=(3.97 \pm 0.01) \times 10^{8} \mathrm{~m}$ and $\alpha=(2.40 \pm 0.02) \times 10^{-8}$ with $\chi_{\min }^{2}=21.47$. 


\section{Conclusions}

In this work, we use 4 double pulsars data (PSR B1913+16, PSR B1534+12, PSR J0737-3039, PSR B2127+11C) to constrain the Yukawa parameters and obtain $\lambda=$ $(3.97 \pm 0.01) \times 10^{8} \mathrm{~m}$ and $\alpha=(2.40 \pm 0.02) \times 10^{-8}$ at $1 \sigma$ level. It demonstrates double pulsars could be a good test-bed for testing gravitational theories.

\section{References}

Anderson, J. D., Laing, P. A., Lau, E. L., et al. 1998, Phys. Rev. Lett., 81, 2858

Bell, J. F., Camilo, F., \& Damour, T. 1996, ApJ, 464, 857

Brownstein, J. R. \& Moffat, J. W. 2006, Classical Quantum Gravity, 23, 3427

Damour, T. \& Esposito-Farèse, G. 1996, Phys. Rev. D, 53, 5541

Detweiler, S. L. 1979, ApJ, 234, 1100

Fischbach, E., Sudarsky, D., Szafer, A. et al. 1986, Phys. Rev. Lett., 56, 3

Fischbach, E. \& Talmadge, C. L. 1999, The search for non-newtonian gravity (Springer-Verlag, New York).

Hulse, R. A., Taylor, J. H. 1975, ApJ, 195, L51 .

Iorio, L. 2007, Planet. Space Sci., 55, 1290

Iorio, L. 2008, arXiv:0809.3563

Kozai, Y. 1959, AJ, 64, 367

Lommen, A. N. \& Backer, D. C. 2001, ApJ 562, 297

Lorimer, D. R. 2005, Living Rev. Relativity, 8, 7

Reynaud, S. \& Jaekel, M. T. 2005, Int. J. Mod. Phys. A, 20, 2294

Sealfon, C., Verde, L., \& Jimenez, R. 2005, Phys. Rev. D, 71, 083004

Stairs, I. H. 2004, Science, 304, 547. 